\title{
Significance of Stabilometry for Assessing Postoperative Body Sway in Patients with Cervical Myelopathy
}

\author{
Shinji Tanishima ${ }^{1}$, Hideki Nagashima ${ }^{1}$, Hiroyuki Ishii ${ }^{2}$, Satoru Fukata ${ }^{2}$, \\ Toshiyuki Dokai ${ }^{3}$, Taiki Murakami ${ }^{1}$,Yasuo Morio ${ }^{2}$ \\ ${ }^{1}$ Orthopedic Surgery, Department of Orthopedic Surgery, Faculty of Medicine, Tottori University, Tottori, Japan \\ ${ }^{2}$ Orthopedic Surgery, Department of Orthopaedic Surgery, Misasa Onsen Hospital, Tottori, Japan \\ ${ }^{3}$ Orthopedic Surgery, Department of Orthopedic Surgery, Masuda Redcross Hospital, Tottori, Japan
}

\section{Study Design: Prospective study.}

Purpose: To examine the changes in body sway using stabilometry in patients who underwent cervical laminoplasty for cervical myelopathy.

Overview of Literature: Although the patients of cervical myelopathy complain body sway there are few report to examine body sway objectively.

Methods: Patients who received treatment for cervical myelopathy between October 2010 and February 2013 were included. Twentyone patients underwent cervical laminoplasty (myelopathy group). Body sway was assessed using stabilometry, wherein patients stood on a stabilometer with their eyes closed for 30 seconds. The Romberg ratio, outer peripheral area (OPA) with eyes closed ( $\left.\mathrm{cm}^{2}\right)$, and total locus length per unit area (L/A) with eyes closed $(/ \mathrm{cm})$ were examined. Examinations were performed preoperatively (at baseline) and at 8 weeks postoperatively. Examination results of patients in the myelopathy group were compared with those of 17 healthy individuals (control group). Clinical symptoms were evaluated using the Japanese Orthopaedic Association scale score (JOA score) and the timed up and go (TUG) test.

Results: In the myelopathy and control groups, the mean baseline Romberg ratio, OPA, and L/A were $2.3 \pm 1.2,8.9 \pm 5.5 \mathrm{~cm}^{2}$, and $14.2 \pm 5.3 / \mathrm{cm}$ and $1.4 \pm 1.0,4.3 \pm 2.8 \mathrm{~cm}^{2}$, and $23.7 \pm 10.1 / \mathrm{cm}$, respectively. Eight weeks after laminoplasty, only L/A showed significant improvement from baseline in the myelopathy group $(23.2 \pm 10.1$ to $16.8 \pm 7.9 ; p=0.03)$. The Romberg ratio and OPA showed improvement in the myelopathy group, but the changes were not statistically significant. JOA scores and TUG test results in this group significantly improved from baseline to 8 weeks after laminoplasty (12.7 to 13.4 and 10.8 to 8.0 seconds, respectively; both $p<0.05$ ). Conclusions: $\mathrm{L} / \mathrm{A}$ is a useful parameter for measuring body sway to assess the recovery of body sway after laminoplasty.

Keywords: Spinal cord diseases; Spine; Postural balance; Laminoplasty

Received Oct 31, 2016; Revised Mar 5, 2017; Accepted Mar 19, 2017

Corresponding author: Shinji Tanishima

Department of Orthopedic Surgery, Faculty of Medicine, Tottori University Hospital, 36-1 Nishi-cho, Yonago, Tottori 683-8504, Japan Tel: +81-859-38-6587, Fax: +81-859-38-658, E-mail: shinjit@med.tottori-u.ac.jp 


\section{Introduction}

Body sway is a symptom of cervical myelopathy. Cervical myelopathy is often caused by spondylosis or the ossification of the posterior longitudinal ligament and is characterized by compression of the cervical spinal cord by varying degrees and at different levels. When the dorsal column of the spinal cord is compressed, the functions of vibration sense, deep sensibility, and joint position sense are lost [1]. This situation impairs the body's sense of balance and results in dysfunction of the upper and lower extremities. Therefore, patients complain of body sway and difficulty in moving their extremities. It is well-known that patients with cervical myelopathy have impaired proprioception abilities [1-4]. To assess impaired upper extremity proprioception, patients with myelopathic hands can be objectively evaluated using the 10 -seconds test and hand function test [3]. However, body sway cannot be objectively measured, and quantitative evaluation is difficult. The 10-seconds step test is the only method to assess lower extremity impairment due to myelopathy $[5,6]$. Postural control mechanisms are analyzed by measuring shifts in the center of gravity using stabilometry $[7,8]$. Stabilometry evaluates a patient's postural control and is used to investigate body sway in several diseases $[9,10]$. Yoshikawa et al. [11] used a stabilometer to investigate the postural stability of patients with cervical myelopathy and reported that a stabilometer can objectively evaluate postural stability, which may reflect the function of the dorsal spinal column.

We considered that stabilometry can be used to assess the recovery of postural balance in patients who underwent laminoplasty. Although a stabilometer can investigate several factors, few reports have specified which parameters are useful in assessing body sway among patients with cervical myelopathy. Yoshikawa et al. [11] showed that stabilometry could be used to objectively evaluate the postural stability of patients with cervical myelopathy based on the environmental area and locus length per area, which are parameters of body sway. Nardone et al. [12] used center of foot pressure and length of the path traveled by the center of foot pressure as parameters of body sway in patients with cervical myelopathy.

In the present study, we hypothesized that body sway can be quantitatively evaluated in patients with cervical myelopathy who underwent laminoplasty. We investigated body sway using a stabilometer in patients with cervical myelopathy before and after laminoplasty.

\section{Materials and Methods}

\section{Subjects}

Patients who underwent laminoplasty for cervical myelopathy between October 2010 and February 2013 at Misasa Onsen Hospital were included. They had clinical symptoms of myelopathy in the upper or lower limbs. All patients presented with signs of mechanical compression of the spinal cord, which was identified using magnetic resonance imaging or myelography, and all had the ability to stand unaided with eyes closed for $>30$ seconds.

Exclusion criteria were pathology in the lower limbs or lumbar spine, history of cerebral infarction, peripheral nerve disorder, and vertigo associated with conditions such as Meniere's disease. Patients who were unable to stand unaided for $>30$ seconds were also excluded.

Twenty-one patients with cervical myelopathy (15 men and 6 women) were enrolled. The mean age of the patients was 68.2 years (range, $50-85$ years). Cervical myelopathy

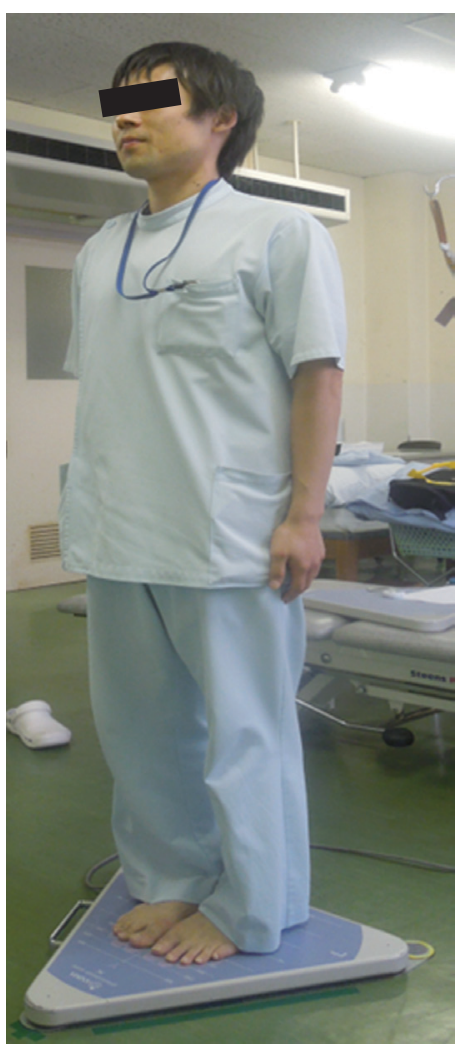

Fig. 1. Patients standing on the stabilometry platform for 30 seconds with their eyes closed. 

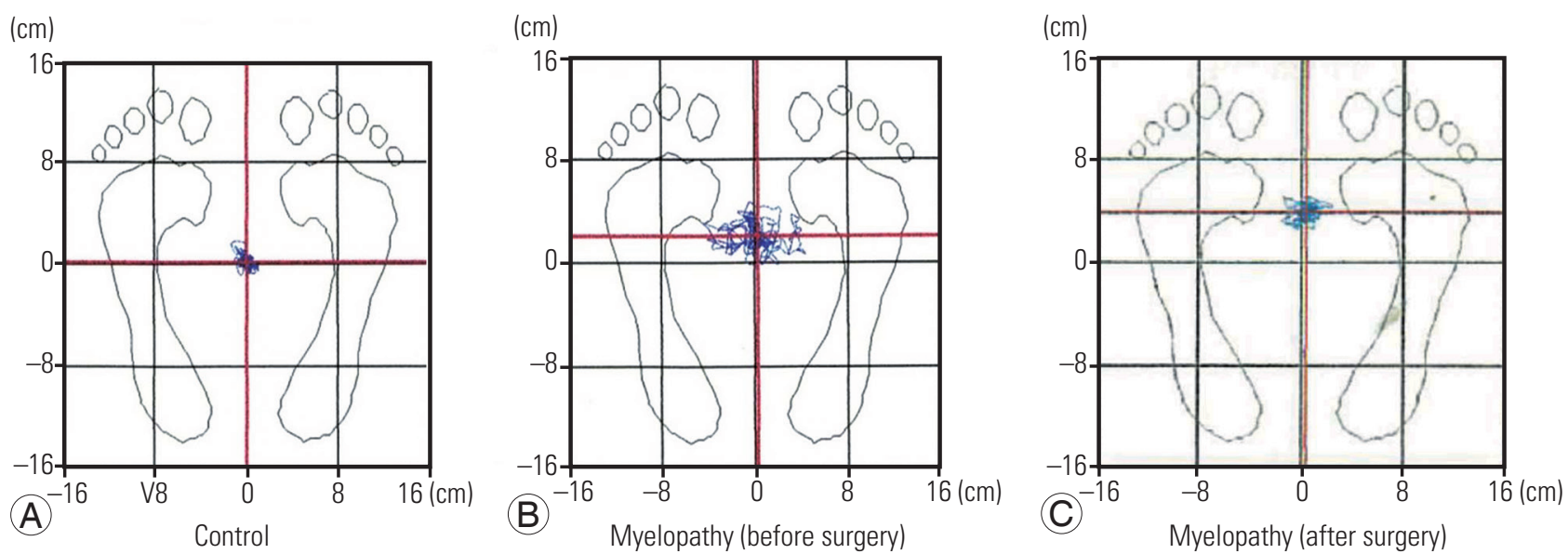

Fig. 2. Sample stabilograms of patients in the control and myelopathy groups. (A) Control group. (B) Myelopathy group preoperatively. (C) Myelopathy group postoperatively.

was caused by cervical spondylosis in 16 patients, ossification of the posterior longitudinal ligament in four, and disc herniation in one; these patients were included in the myelopathy group. They were compared with a control group consisting of 17 age-matched, normal, healthy volunteers (1 man and 16 women), with an average age of 71.9 years (range, $61-82$ years), who had no abnormalities in either the lower limbs or spine. All the participants provided written informed consent, and this study was approved by the local ethics committees of the Misasa Onsen Hospital.

\section{Measurement procedures}

The stabilometer used was Gravicorder G-620 (Anima Corporation, Tokyo, Japan). Patients stood on the stabilometry platform for 30 seconds with their eyes closed (Fig. 1).

The sway of the center of gravity was measured using the standard method. Each patient stood barefoot on the platform in an upright position, with both feet together and both eyes closed for 30 seconds. This assessment was performed preoperatively (baseline) and at 8 weeks postoperatively. Data were analyzed using static stabilogram analysis software provided with the Gravicorder G-620, and the values obtained were recorded. Typical examples of the stabilograms from the control and myelopathy groups are shown Fig. 2. The stabilogram shows the track of the center of gravity.

Three parameters were used for evaluation (Fig. 3).

(1) Romberg ratio: An index of the dependency on vision for postural control during quiet stance. (2) Outer

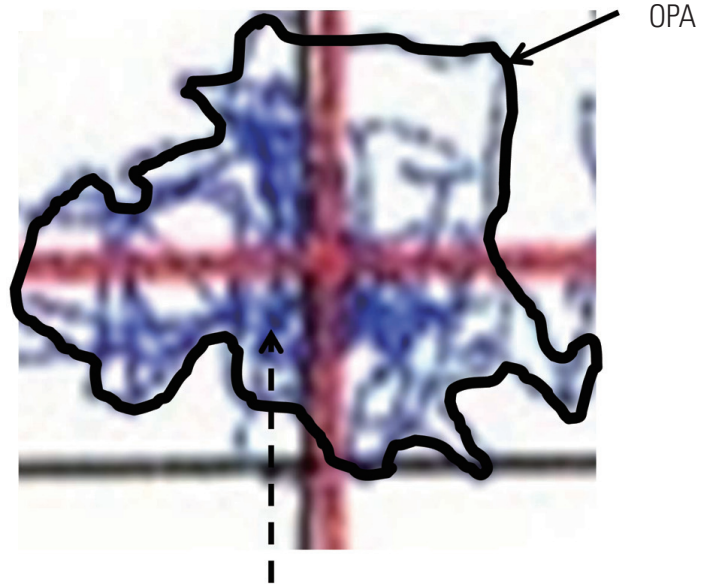

Total locus length

Fig. 3. Stabilogram parameters used for evaluation. OPA, outer peripheral area with the track of the center of gravity (black arrow); Total locus length, length of the center of gravity (dashed arrow pointed blue line); L/A, total locus length/OPA; Romberg ratio, OPA (eye closed)/ OPA (eye opened).

peripheral area (OPA): the area that encloses the circumference of the stabilogram, which measures the degree of sway from the center of gravity. (3) Total locus length per environmental area (L/A): This parameter measures the fine control of the standing posture by the proprioceptive reflexes.

\section{Clinical assessment}

Clinical results were evaluated based on motor and sensory function testing using the Japanese Orthopaedic Association (JOA) scale (total, 17 points) and the timed up and go (TUG) test. The TUG test requires patients to stand up 
Table 1. Stabilometry results in the control and myelopathy groups

\begin{tabular}{lccc} 
Parameters & Control group & Myelopathy group & $p$-value \\
Romberg ratio & $1.4 \pm 1.0$ & $2.3 \pm 1.2$ & 0.020 \\
OPA $\left(\mathrm{cm}^{2}\right)$ & $4.3 \pm 2.8$ & $8.9 \pm 5.5$ & 0.003 \\
L/A $(/ \mathrm{cm})$ & $23.2 \pm 10.1$ & $14.2 \pm 5.3$ & 0.001 \\
\hline
\end{tabular}

Values are presented as mean \pm standard deviation.

OPA, outer peripheral area; L/A, total locus length per environmental area.

a) Student's $t$-test.

from a chair, walk a short distance, turn around, return, and sit down on the chair again. We performed this test according to the procedure proposed by Mathias et al. [13]. JOA scores were assessed by a physician preoperatively and at 4 and 8 weeks after laminoplasty. The TUG test was performed by a physical therapist at 4 and 8 weeks after laminoplasty.

\section{Statistical analysis}

The unpaired $t$-test was used to evaluate the differences in the Romberg ratio, OPA, and L/A between the patients in the myelopathy and control groups. The Student's $t$-test was used to evaluate the differences in the JOA scores and TUG test results obtained before and after laminoplasty. The level of statistical significance was set at 5\% $(p<0.05)$ for all tests. StatMate for Windows ver. 4.01 (ATMS Corporation, Tokyo, Japan) was used for all statistical calculations.

\section{Results}

None of the patients experienced any surgical complications.

\section{Comparison of stabilogram measurements between the patients in the myelopathy and control groups}

At baseline, the mean Romberg ratios in the myelopathy and control groups were $2.3 \pm 1.2$ and $1.4 \pm 1.0$, respectively $(p=0.020)$. The mean OPAs in the myelopathy and control groups were $8.9 \pm 5.5 \mathrm{~cm}^{2}$ and $4.3 \pm 2.8 \mathrm{~cm}^{2}$, respectively ( $p=0.003$ ), and the mean L/As were $14.2 \pm 5.3 / \mathrm{cm}$ and $23.7 \pm 10.1 / \mathrm{cm}$, respectively $(p=0.001)$ (Table 1$)$.

\section{Clinical results: JOA scores and TUG findings}

In the myelopathy group, the mean JOA scores improved
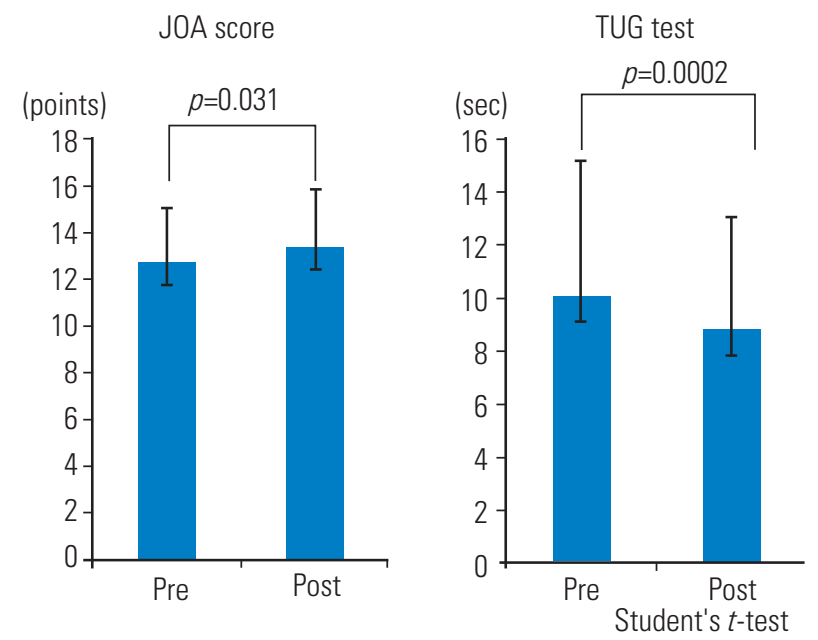

Fig. 4. A comparison of the JOA scores and TUG test results pre and post laminoplasty in the myelopathy group. JOA, Japanese Orthopaedic Association; TUG, timed up and go.

from $12.7 \pm 2.4$ at baseline to $13.4 \pm 2.5$ at 8 weeks after laminoplasty. The TUG test results improved from 10.1 \pm 5.1 seconds at baseline to $8.0 \pm 3.4$ seconds at 8 weeks after surgery Both changes were statistically significant $(p<0.05)$ (Fig. 4).

\section{Pre- and post-laminoplasty stabilogram results in the myelopathy group}

In the myelopathy group, the mean Romberg ratio changed from $2.3 \pm 1.2$ to $2.1 \pm 1.2$, and the mean OPA decreased from $8.9 \pm 5.5 \mathrm{~cm}^{2}$ at baseline to $7.9 \pm 4.5 \mathrm{~cm}^{2}$ at 8 weeks after laminoplasty. These changes were not statistically significant. The mean L/A significantly improved from $18.3 \pm 5.0 / \mathrm{cm}$ at baseline to $16.8 \pm 7.7 / \mathrm{cm}$ at 8 weeks after laminoplasty $(p<0.03)$ (Table 2$)$.

\section{Discussion}

Body stability when standing upright depends on the vestibular, visual, and somatosensory systems [14]. These 
Table 2. The comparison of the stabilometry results pre and post laminoplasty in the myelopathy group

\begin{tabular}{lccc} 
Parameters & Pre & Post & $p$-value \\
Romberg ratio & $2.3 \pm 1.2$ & $2.1 \pm 1.2$ & 0.32 \\
OPA $\left(\mathrm{cm}^{2}\right)$ & $8.9 \pm 5.5$ & $7.9 \pm 4.6$ & 0.19 \\
L/A $/(\mathrm{cm})$ & $23.2 \pm 10.1$ & $16.8 \pm 7.9$ & 0.03 \\
\hline
\end{tabular}

Values are presented as mean \pm standard deviation.

OPA, outer peripheral area (the area that encloses the circumference of the stabilogram, which measures the degree of sway from the centre of gravity); L/A, total locus length per environmental area (this measures fine control of the standing posture by proprioceptive reflexes).

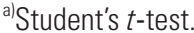

systems contribute to the maintenance of postural control. The spinal cord, particularly the dorsal column, plays an important role as the somatosensory system [15]. Ross [16] have mentioned that the dorsal column controls the position and vibration sensations and plays an important role in maintaining postural stability and conveying sensory information such as deep sensations to the lower limbs. When the dorsal column of the spinal cord is compressed, the functions of vibration sense, deep sensibility, and joint position sense are lost. Takayama et al. [1] used an electrogoniometer to demonstrate that patients with cervical myelopathy have impaired knee proprioception. A damaged spinal cord causes impaired body balance because of proprioceptive loss, and patients develop ataxia in the lower limbs. This mechanism explains why patients with myelopathy develop body sway.

Body sway is a subjective symptom associated with cervical myelopathy. It can be objectively observed, but it is difficult to quantitatively assess. Various investigations have been performed to assess body sway. Gill et al. [17] measured body sway in normal subjects using angular velocity transducers worn at the waist and showed their usefulness in screening balance disorders. Overstall et al. [18] investigated body sway in elderly women using an ataxiameter and suggested that elderly women had poor postural control. Although these methods are useful, the procedures are not simple. In this study, we used stabilometry, which is easy to use for quantitatively measuring body sway. Terekhov [19] measured body sway in patients with cervical or spinal tumors using stabilometry; he described the efficacy and simplicity of investigating the stability of stance and related it to both the physiology of the central nervous system and medical cybernetics. Yoshikawa et al. [11] assessed patients with cervical myelopathy using a stabilometer. Their results indicated that two parameters of postural instability were correlated with clinical symptoms in patients with myelopathy.
To the best of our knowledge, the present study is the first to evaluate the recovery of body balance postoperatively using stabilometry. The Romberg ratio, OPA, and L/ $A$ are useful parameters to evaluate equilibrium, and we assessed body sway using these three parameters in this study.

At baseline, the myelopathy and control groups showed a significant difference in these parameters. In the myelopathy group, the Romberg ratio and OPA did not show significant improvement from baseline to 8 weeks after laminoplasty. Nevertheless, this group showed good improvement in the TUG test results and JOA scores. In particular, we considered that improvement in the TUG test results reflected the patients' recovery from cervical myelopathy. These results indicated that surgery was a good treatment option for this condition. Although the clinical results indicated good recovery, the Romberg ratio and OPA did not improve postoperatively.

The Romberg ratio did not show significant improvement from baseline, but previous studies have shown that stabilometry has good relative and satisfactory absolute intraobserver reliability [20,21]. Tjernstrom et al. [22] investigated the Romberg ratio using posturography in normal subjects. The Romberg ratio exhibited considerable intraindividual variability because it is easily affected by visual field dependence. We considered that this ratio may yield variable results. In addition, our study duration was a relatively short period of 8 weeks. It is possible that 8 weeks are not adequate to assess the evolution of body balance using stabilometry. In this study, there was no significant difference in the Romberg ratio and OPA from baseline to 8 weeks after laminoplasty in the myelopathy group.

OPA is one of the parameters quantifying body sway. Similar to the Romberg ratio, OPA did not significantly improve from baseline to 8 weeks after laminoplasty in the myelopathy group. OPA usually shows the degree of 
sway of the center of gravity similar to the Romberg ratio. Therefore, the Romberg ratio and OPA may not be ideal for assessing the recovery in body sway at such an early stage postoperatively. Only the patients' clinical symptoms and L/A showed significant improvement after laminoplasty. L/A indicates the standing postural control ability in such cases. Akabane et al. [23] reported that they assessed body sway after an epidural block. L/A was a more useful evaluation parameter than the Romberg ratio. We consider that L/A could reflect recovery postoperatively.

We consider that stabilometry can be used to assess the recovery of patients with myelopathy postoperatively; in particular, L/A is a useful parameter for assessing postural stability in the early phase postoperatively.

This study has some limitations. First, we assessed only patients who could stand by themselves without any assistance. Second, we could not assess patients with severe myelopathy. Other procedures should be used to assess body sway in such patients. Third, the study period was 8 weeks. This might have affected the results and may explain why the Romberg ratio and OPA did not improve.

\section{Conclusions}

Stabilometry reflects the body sway in patients with cervical myelopathy and can be used to quantitatively assess recovery after laminoplasty. L/A is a useful parameter for assessing body sway in patients with cervical myelopathy in the early stage postoperatively.

\section{Conflict of Interest}

No potential conflict of interest relevant to this article was reported.

\section{References}

1. Takayama H, Muratsu H, Doita M, Harada T, Yoshiya S, Kurosaka M. Impaired joint proprioception in patients with cervical myelopathy. Spine (Phila Pa 1976) 2005;30:83-6.

2. Doita M, Sakai H, Harada T, et al. The influence of proprioceptive impairment on hand function in patients with cervical myelopathy. Spine (Phila Pa 1976) 2006;31:1580-4.

3. Doita M, Sakai H, Harada T, et al. Evaluation of impairment of hand function in patients with cervical myelopathy. J Spinal Disord Tech 2006;19:276-80.

4. Takayama H, Muratsu H, Doita M, Harada T, Kurosaka M, Yoshiya S. Proprioceptive recovery of patients with cervical myelopathy after surgical decompression. Spine (Phila Pa 1976) 2005;30:1039-44.

5. Yukawa Y, Kato F, Ito K, et al. "Ten second step test" as a new quantifiable parameter of cervical myelopathy. Spine (Phila Pa 1976) 2009;34:82-6.

6. Ogawa Y, Yukawa Y, Morita D, Ito K, Machino M, Kato F. 10-second step test for quantitative evaluation of the severity of thoracic compressive myelopathy. Spine (Phila Pa 1976) 2013;38:1405-8.

7. Kapteyn TS, Bles W, Njiokiktjien CJ, Kodde L, Massen CH, Mol JM. Standardization in platform stabilometry being a part of posturography. Agressologie 1983;24:321-6.

8. Fujita T, Nakamura S, Ohue M, et al. Effect of age on body sway assessed by computerized posturography. J Bone Miner Metab 2005;23:152-6.

9. Rossi C, Alberti A, Sarchielli P, et al. Balance disorders in headache patients: evaluation by computerized static stabilometry. Acta Neurol Scand 2005;111:407-13.

10. Ohashi N, Nakagawa H, Asai M. Contribution of vision to the stabilization of body sway in patients with spinocerebellar degeneration. Acta Otolaryngol Suppl 1993;504:117-9.

11. Yoshikawa M, Doita M, Okamoto K, Manabe M, Sha N, Kurosaka M. Impaired postural stability in patients with cervical myelopathy: evaluation by computerized static stabilometry. Spine (Phila Pa 1976) 2008;33:E460-4.

12. Nardone A, Galante M, Grasso M, Schieppati M. Stance ataxia and delayed leg muscle responses to postural perturbations in cervical spondylotic myelopathy. J Rehabil Med 2008;40:539-47.

13. Mathias S, Nayak US, Isaacs B. Balance in elderly patients: the "get-up and go" test. Arch Phys Med Rehabil 1986;67:387-9.

14. Allum JH, Pfaltz CR. Visual and vestibular contributions to pitch sway stabilization in the ankle muscles of normals and patients with bilateral peripheral vestibular deficits. Exp Brain Res 1985;58:82-94.

15. Wall PD, Noordenbos W. Sensory functions which remain in man after complete transection of dorsal columns. Brain 1977;100:641-53.

16. Ross RT. Dissociated loss of vibration, joint position 
and discriminatory tactile senses in disease of spinal cord and brain. Can J Neurol Sci 1991;18:312-20.

17. Gill J, Allum JH, Carpenter MG, et al. Trunk sway measures of postural stability during clinical balance tests: effects of age. J Gerontol A Biol Sci Med Sci 2001;56:M438-47.

18. Overstall PW, Exton-Smith AN, Imms FJ, Johnson AL. Falls in the elderly related to postural imbalance. Br Med J 1977;1:261-4.

19. Terekhov Y. Stabilometry as a diagnostic tool in clinical medicine. Can Med Assoc J 1976;115:631-3.

20. Schwesig R, Fischer D, Becker S, Lauenroth A.
Intraobserver reliability of posturography in patients with vestibular neuritis. Somatosens Mot Res 2014;31:28-34.

21. Schwesig R, Becker S, Fischer D. Intraobserver reliability of posturography in healthy subjects. Somatosens Mot Res 2014;31:16-22.

22. Tjernstrom F, Bjorklund M, Malmstrom EM. Romberg ratio in quiet stance posturography: test to retest reliability. Gait Posture 2015;42:27-31.

23. Akabane H, Shimada Y, Ogawa R. Usefulness of posturography after epidural block. J Nippon Med Sch 2004;71:35-43. 